\title{
Identification of 11 STD Pathogens in Semen Using Polymerase Chain Reaction (PCR) and "Flow-through" Hybridization Technology
}

\author{
Rubina Ghani ${ }^{1}$, Kashif Nisar ${ }^{1}$, Hasan $_{\mathrm{Ali}^{2}}$ and Saara $\mathrm{Ahmad}^{3}$ \\ 1. Department of Biochemistry, Jinnah Medical \& Dental College, Pathological \& Molecular Laboratories Karachi, Pakistan \\ 2. Department of Biochemistry, Bahria University, Bahria Medical \& Dental College \\ 3. Department of Biochemistry, Baqai Medical University, Pathological \& Molecular Laboratories Karachi, Pakistan
}

\begin{abstract}
The transmission of sexually transmitted infection (STI) pathogens from an infected donor to the recipient of a semen donation in assisted conception may result not only in acute infection but also in long-term reproductive complications or adverse outcomes of pregnancy including infection of the offspring. Semen samples were obtained by masturbation into sterile containers. Samples were subjected to semen analysis within one hour of collection and processed for freezing within two hours of collection. The sperm motility was determined. After DNA extraction the PCR was performed and amplicons are subsequently hybridized to pathogen-specific capturing probes via "Flow-through" hybridization. During our study we came across with the STI pathogens present in semen and main cause of infertility was note. It was also observed that route for the transfer for these STI pathogens were the men working in other cities and visited commercial sex workers and their complained for infertility. We have reported our data that after the normal sperm count in semen samples of men with infertility or subfertility they were infected with Chlamtdia trachomatis, Neisseria gonorrhoeae, and Mycoplasma hominis. In our study all the 11 pathogens were detected which cause serious reproductive complications and infection in their offspring.
\end{abstract}

Key words: Sexually transmitted disease, semen analysiss, chlamydia trachomatis, Neisseria gonorrhoeae, Mycoplasma hominis, Human Pappilloma virus.

\section{Introduction}

A variety of sexually transmitted infectious (STIs) of the male genitourinary (GU) tract have been associated with male factor infertility. In both men and women these infections may result in significant morbidity and make a formidable contribution to world-wide health expenditure [1-5]. Infertility is the failure of a couple to become pregnant after one year of regular, unprotected intercourse. About a third of infertility problems are due to female infertility, and another third are due to male infertility. In the remaining cases, infertility affects both partners or the cause is unclear [6]. Sexually transmitted infections (STIs) are recognized as a major public health

Corresponding author: Rubina Ghani, M.Sc., Ph.D., research fields: infectious disease and molecular diagnosis. problem with severe medical and psychological consequences for millions of men and women [7]. The number of STIs is increasing throughout the world despite diagnostic and treatment advances in recent years [8].

More than 340 million cases of curable sexually transmitted infections (STIs) including Treponema pallidum, Neisseria gonorrhoeae, Chlamydia trachomatis and Trichomonas vaginalis were estimated to have occurred worldwide in 1995 [9]. Previous studies have shown that the presence of other concomitant STIs increases the likelihood of HIV transmission [10-13].

As in other developing countries, sexually transmitted infections (STIs) and reproductive tract infections (RTIs) represent a major public health problem in Bangladesh [6]. Control of ulcerative 
(syphilis, chancroid, and herpes simplex virus type 2 [HSV-2] infection) and nonulcerative (gonorrhea, chlamydia, and trichomoniasis) STIs and of RTIs (bacterial vaginosis and candidiasis) is important not only for preventing complications related to infection but also for preventing heterosexual transmission of human immunodeficiency virus (HIV).

STDs are not only a cause of acute morbidity in adults but may result in complications with sequelae such as infertility in both men and women, ectopic pregnancy, cervical cancer, premature mortality, congenital syphilis and fetal wastage, low birth weight, and prematurity and ophthalmia neonatorum. The STDs that are caused by bacterial, mycological and protozoal agents have been curable by appropriate antibiotics and chemotherapeutic agents for more than 40 years. In spite of this, such STDs have continued to be a public health problem in both industrialized and developing countries. In many developing countries STDs have for several decades ranked among the top five diseases for which adults seek health care services. Reliable surveillance is rarely in place and the exact magnitude of the problem is frequently unknown.

The goal of estimating correctly a man's fertility potential has long been of great interest to researchers. The term 'male infertility' does not constitute a defined clinical syndrome but rather a collection of different conditions exhibiting a variety of etiologies and varying prognoses [6].

The present guidelines are intended for laboratories involved in the testing of semen samples to ensure, within the limitations of existing laboratory methods, that the semen samples are free from pathogens that can cause sexually transmitted infections (STIs). Testing of semen specimens for STI pathogens is not recommended as a means of diagnosis of clinical syndromes in donors of assisted conception programs, nor should these specimens be used in tests of cure following treatment. Donors generally undergo vigorous screening for STIs before they are accepted into a semen donation program [14-18].

The objective of screening semen samples before they are used for assisted reproduction procedures is to protect the recipients of semen donations and their offspring from bacterial and viral STIs and their sequelae by preventing the transmission of STI pathogens from them to the recipient (partners). The transmission of STI pathogens to the recipient of a semen donation may result not only in acute infection, but also in long-term reproductive complications in the recipient and possible adverse outcomes of pregnancy, including infection in the offspring [19-21].

\section{Material and Method}

\subsection{Specimen Collection}

The duration of this study was for two months i.e. from November2013 to January 2014 and all the semen samples were collected with history and cause of infertility. During this study, the history form was filled and we found that most of the man were driver and their family were settle in small village or Goth areas people settle in Karachi not only came from four provinces but from sample villages in Sindh for jobs.

In this study samples were selected from different clinics, maternity homes and medical centers from different parts of Karachi. As Karachi is the cosmopolitan city and all the people from different parts of country come here for the business and its economy is based on industrial production, trade and finance. As well as it is the commercial and industrial capital of the country.

The semen sample was distributed into four parts and send to each department for the basic test. The serology, culture and routine semen analysis test were performed and data was collected, whereas with one part of the sample was used for the DNA extraction.

DNA extraction: The DNA purification from whole blood collected in EDTA was carried out by using Epicenter Kit (cat\# MCD85201) and the protocol was followed accordingly. 


\subsection{PCR Amplification and Gene Flow Hybridization}

The PCR was performed. The Amplification Control (signal at B5) is used to monitor the presence of PCR inhibitors and the presence of sufficient amount of extracted human cellular DNA content during PCR amplification. Absence of Amplification Control signal indicates either failure of the PCR amplification (presence of PCR inhibitor) or insufficient amount of DNA (or absence of specimen) during PCR amplification. This control signal should always be positive with a clear visible dot in every tested sample.

The PCR reaction was prepared according to protocol given table 1 and the final volume was made upto $25 \mu \mathrm{L}$.

Aliquot $20 \mu \mathrm{L}$ of Master Mix (containing PCR Reaction Mix, 25X Primer Mix and DNA Taq Polymerase) into each PCR tube and add appropriate amount of DNA template suggested. Top up the reaction volume to $25 \mu \mathrm{L}$ with DNase Free Water when necessary. Mix and spin down the PCR reaction mixture (including the sample). PCR tubes were placed into thermal cycler and run following the thermal condition in table 2 below

After amplification the sample tubes were cooled and were prepared for the hybridization following steps given in the table 3 .

\section{Result}

The 60 semen analysis samples were collected from $\mathrm{a}$ lab and the basic screening was done in which sperm morphology and its motility was checked according to WHO guide line as shown in table 4.

Then the PCR was performed on those samples whose count was normal and there was infertility. The

Table 1 Shows the protocol to make master mix for PCR amplification.

\begin{tabular}{ll}
\hline Component & Volume $(\mu \mathrm{l})$ \\
\hline PCR Reaction Mix & 18.6 \\
25X Primer Mix & 1.0 \\
DNA Taq Polymerase & 0.4 \\
DNA Template & Up to $5.0 \mu \mathrm{L}$ \\
DNase Free Water & Variable \\
Total & 25.0 \\
\hline
\end{tabular}

Table 2 Shows the thermal cycle condition according to which the DNA is amplified.

\begin{tabular}{llll}
\hline Stage & Step & Temperature $\left({ }^{\circ} \mathrm{C}\right)$ & Time \\
\hline Hold & Initial denaturation & 95 & $10 \mathrm{~min}$ \\
& Denaturation & 95 & $30 \mathrm{sec}$ \\
43 cycles & Annealing & 60 & $30 \mathrm{sec}$ \\
& Extension & 72 & $60 \mathrm{sec}$ \\
Hold & Final extension & 72 & $7 \mathrm{~min}$ \\
Hold & Final hold & 4 & $\infty$ \\
\hline
\end{tabular}

Table 3 Shows the protocol for the flow through hybridization at a glance with FT Pro Flow-through.

\begin{tabular}{|c|c|c|c|}
\hline \multicolumn{4}{|c|}{ Hybridization-at-a-glance } \\
\hline Solution & Volume $(\mu \mathrm{l})$ & Incubation & After incubation \\
\hline \multicolumn{4}{|c|}{$43^{\circ} \mathrm{CFT}^{\text {pro }}$} \\
\hline Pre-hybridization & 150 & $2 \min$ & Drain \\
\hline Hybridization & $150+25$ PCR product & $5 \mathrm{~min}$ & Drain \\
\hline Hybridization solution wash & $200 \times 3$ & - & Drain \\
\hline \multicolumn{4}{|c|}{$25^{\circ} \mathrm{C}$} \\
\hline Blocking & 150 & $5 \mathrm{~min}$ & Drain \\
\hline Enzyme Conjugate & 150 & $5 \min$ & Drain \\
\hline \multicolumn{4}{|c|}{$36^{\circ} \mathrm{C}$} \\
\hline Wash A solution & $200 \times 4$ & - & Drain \\
\hline Detection Solution & 150 & $3 \mathrm{~min}$ & Drain \\
\hline Wash A solution & $200 \times 3$ & & Drain \\
\hline Stop & 150 & $1 \mathrm{~min}$ & Drain \\
\hline
\end{tabular}


Table 4 Shows the basic analysis performed on semen samples to filter the sperm morphology.

\begin{tabular}{|c|c|c|c|c|c|}
\hline Motility & Normal & Watery & Viscous & & \\
\hline Viscosity & $\leq 20 \%$ & $\geq 40 \%$ & $\geq 40$ & & \\
\hline \multirow[t]{2}{*}{ Sperm Morphology } & Normal Form & Head Defective & $\begin{array}{l}\text { Mid Piece \& Neck } \\
\text { Defective }\end{array}$ & Tail Defective & $\begin{array}{l}\text { Teratozoo-spermia } \\
\text { index }\end{array}$ \\
\hline & $\leq 20$ & $\geq 30$ & $\geq 20$ & $\geq 10$ & $\geq 2: 1$ \\
\hline \multirow[t]{2}{*}{ Culture / Sensitivity } & Debris & Round cell & Epithelial cells & Erythrocytes & \\
\hline & Moderate or Heavy & $\geq 10 \%$ & $\geq 4 \%$ & Present & \\
\hline Agglutination & $\begin{array}{l}\text { Normal } \\
\leq 10\end{array}$ & $\begin{array}{l}\text { Head to Head } \\
\geq 20\end{array}$ & $\begin{array}{l}\text { Head to Tail } \\
\geq 10\end{array}$ & $\begin{array}{l}\text { Tail to Tail } \\
\geq 10\end{array}$ & \\
\hline
\end{tabular}

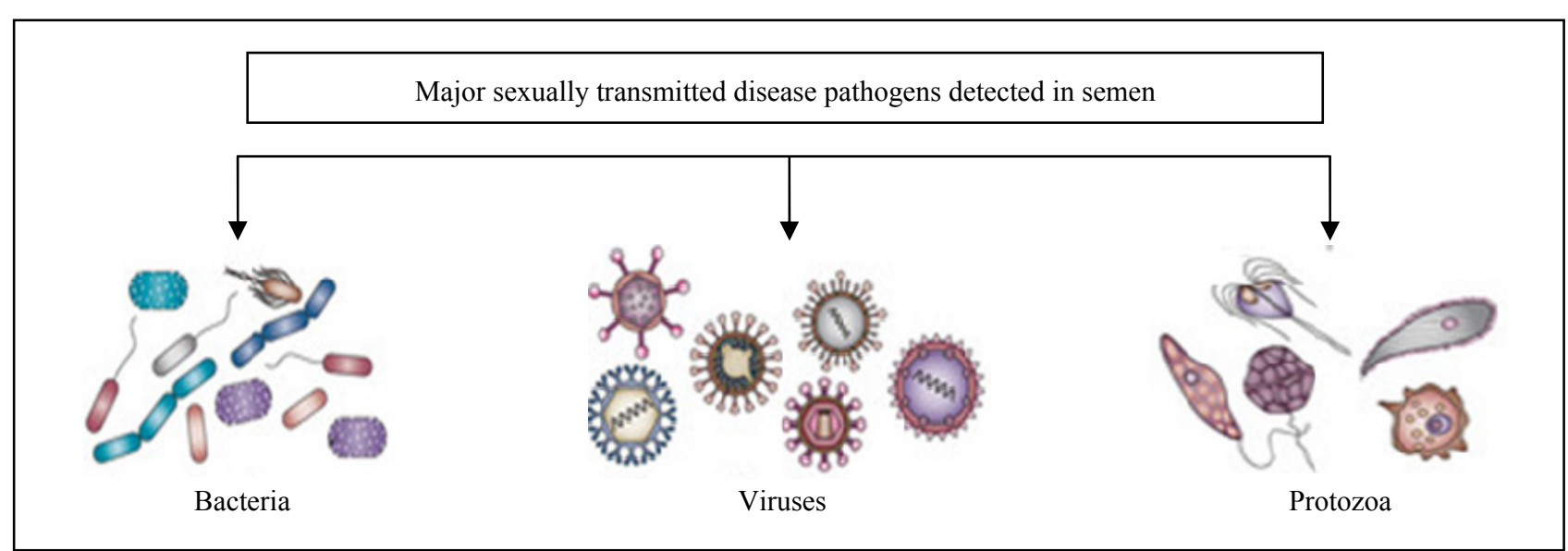

Fig. 1 These are the major sexually transmitted pathogens which are detected in semen.

gene Flow-Through array test was performed and 11 pathogens were detected the semen those causing infertility shown in Fig. 2 (the photograph of cassette). In table 5 the pathogen probes and identification of major STD pathogens causing infertility which were detected in during this study.

When molecular analysis was carried out table 5 was used for the identification of STD probes placed on the cassette. All the 11 pathogens probes can be identified for each patient in one go.

The table 5 shows the position of pathogen probe placed in the membrane on which the amplified product is hybridized with 35 to $40 \mathrm{~min}$.

During our study we detected Chlamydia trachomatis, neisseria gonorrhoeae, mycoplasma genitalium, ureaplasma urealyticum/ureaplasma parvum, mycoplasma hominis, trichomonas vaginalis and human papilliomas virus $6 / 11$ in the semen samples which were having low count and infertility problems detected by gene Flow-through as shown in Fig. 2, which were identified according to the in table 5 .
The different percentage of STD identified in the samples is shown in pie diagram Fig. 3 and table 6.

During our study we also came across with the co-infection which Chlamydia trachomatis, neisseria gonorrhoeae, mycoplasma genitalium, ureaplasma urealyticum/ureaplasma as shown in Fig. 4.

\section{Discussion}

Male factor infertility can be caused by abnormalities in the shape and size of the sperm. As with low sperm count, at least two-thirds of the sperm in the ejaculate must be of an adequate shape and size in order to be considered normal. Male infertility can result when the sperms shape prevents it from having normal mobility or strength to penetrate the ovum. In fact, some specialists believe that abnormal sperm shape has an even greater effect on male fertility than low motility or oligospermia (low sperm count). Some of the causes of abnormal semen, such as STDs, infection, retrograde ejaculation, and the ability of the ejaculate to clot properly, can significantly affect male fertility. 


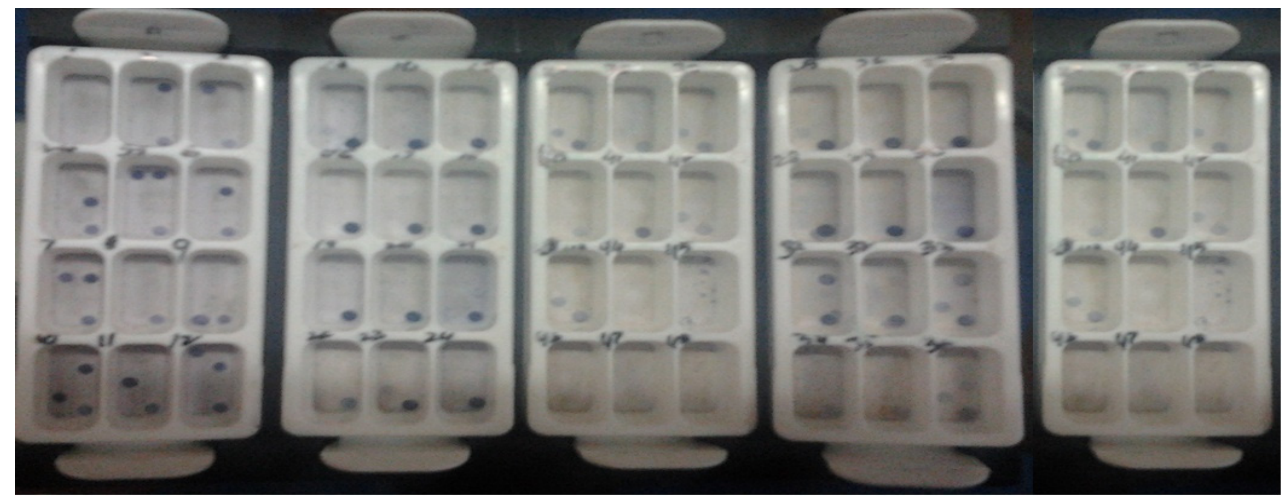

Fig. 2 Shows the target pathogens detected by Gene Flow-Through from semen sample.

Table 5 Shows the identification panel of STD probes placed on membrane.

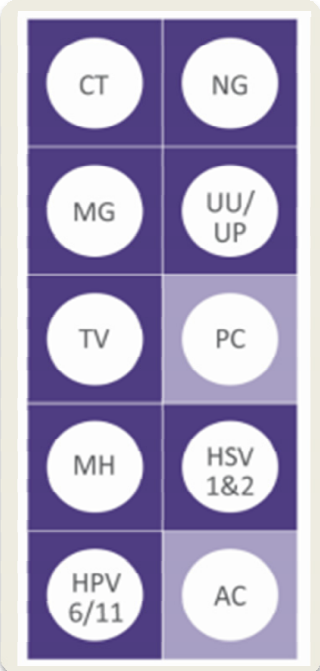

\section{Detect 11 common STD pathogens:}

1. Chlamydia trachomatis (CT)

2. Neisseria gonorrhoeae (NG)

3. Mycoplasma genitalium (MG)

4. Ureaplasma urealyticum (UU)

5. Ureaplasma parvum (UP)

6. Trichomonas vaginalis (TV)

7. Mycoplasma hominis (MH)

8. Human papillomavirus type 6

9. Human papillomavirus type 11

10. Herpes simplex virus type 1

11. Herpes simplex virus type 2

12. Amplification Control (AC)*

13. Positive Control (PC)*

\section{Major STD detected in Semen Samples}

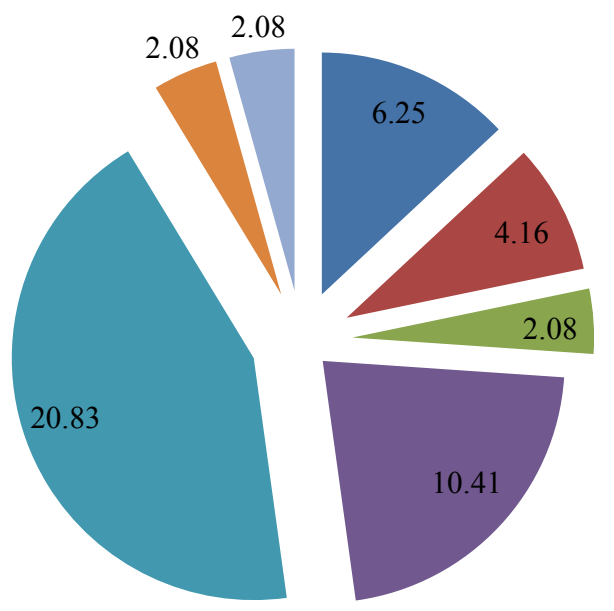

Fig. 3 This shows the percentage of target pathogens detected by Gene Flow-Through from Semen sample. 
Table 6 Shows the different STD detected from semen analysis by Gene Flow-Through to for the cause of infertility.

\begin{tabular}{lll}
\hline NAME OF THE STD $(\mathrm{N}=48)$ & NO. DETECTED & PERCENTAGE \\
\hline Chlamydia trachomatis (CT) & 03 & 6.25 \\
Neisseria gonorrhoeae (NG) & 02 & 4.16 \\
Mycoplasma genitalium (MG) & 01 & 2.08 \\
Ureaplasma Urealyticum (UU)/Ureaplasma parvum (UP) & 05 & 10.41 \\
Human Papillomavirus (HPV) & 01 & 2.08 \\
Mycoplasma hominis (MH) & 10 & 20.83 \\
Trichomonas vaginalis (TV) & 01 & 2.08 \\
Positive Control (PC) & 01 & 2.08 \\
\hline
\end{tabular}

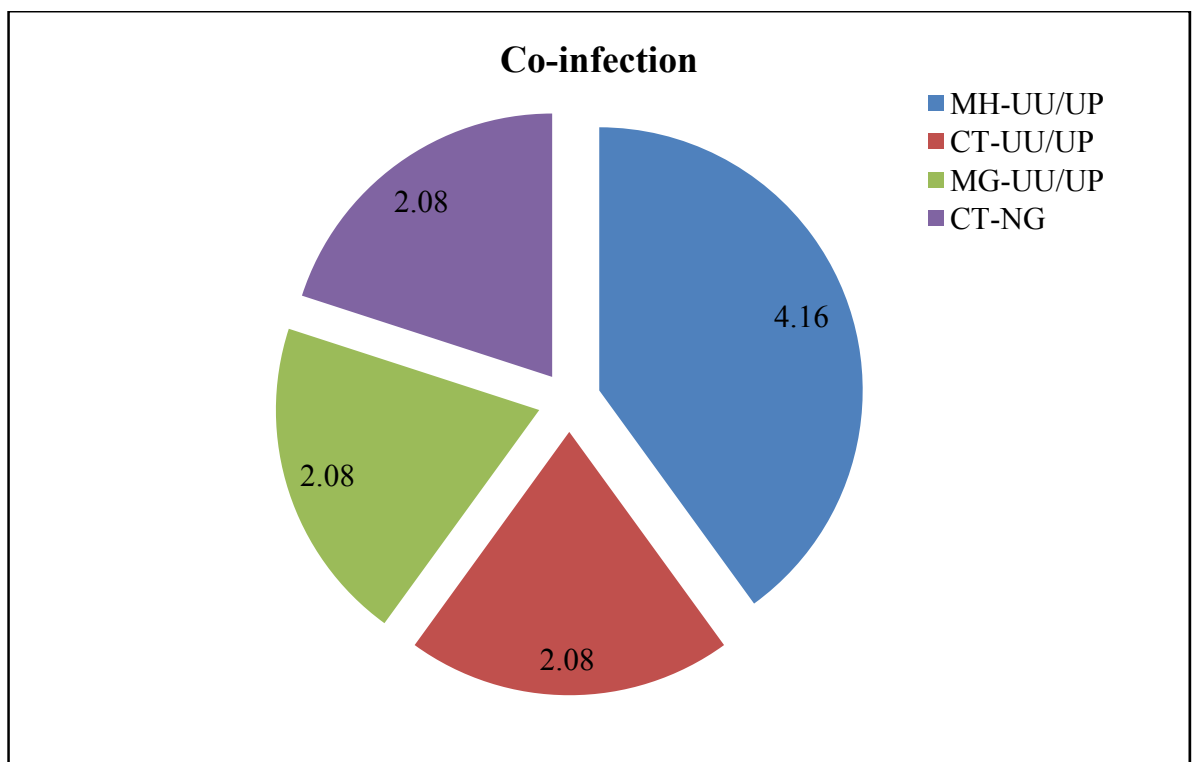

Fig. 4 The distribution pie diagram shows the co-infection rate detected in the semen samples.

Sexual transmission via penile-vaginal, penile-rectal, or penile-pharyngeal contact accounts for the vast majority of cases. The most important means of non-sexual transmission is from mother to child during birth. The gonococcus is a survivor. Despite its being too fragile to be transmitted by food, water, air, or (with very rare exceptions) fomites, it remained a very common infection in most of the world in the preantibiotic era despite sometimes strenuous efforts to control it. The introduction of antimicrobials led to control of gonorrhea in some populations, but despite the natural susceptibility of the gonococci to low concentrations of a wide variety of antimicrobials, 75 years after the discovery of penicillin gonorrhea remains a common infection in much of the world [22].

The demand for assisted reproduction has increased over the last few years because of infertility problems among young couples [23]. We designed our study to investigate the prevalence of 11 pathogens, in the semen of healthy fertile and infertile males, using a sensitive Gene Flow Through technology. Our study showed that genital mycoplasmas and ureaplasmas were the most prevalent uropathogens in the semen of infertile men and were found in asymptomatic fertile men in almost equal proportion, although $\mathrm{M}$ hominis was significantly more prevalent that is 20.83 percent in fertile than infertile men. The second most prevalent microorganism detected was ureaplasma urealyticum/ureaplasma which was 10.41 percent. Male factor infertility in the majority of the cases remains asymptomatic and with unknown causes.

In our clinical studies besides mycoplasma incidence was raised the other pathogen such as Trichomonas vaginalis, Chlamydia trachomatis or 
Neisseria gonorrhea were also detected. Maleki et al. in 2013 [24] reported that strong relation between the presence of the studied $M$. hominis and $U$. urealyticum with urogenital infection in the females under study in comparison with those of control groups.

In previous study, the role of genital tract microorganisms as an important etiological factor in male infertility has been reported [25]. Whereas Friberg and Gnarpe in 1974 had earlier demonstrated the preponderance of ureaplasmas in the semen of infertile men (76\%) compared with fertile men (19\%) [26].

In many literature reports, $\mathrm{U}$ urealyticum and $\mathrm{C}$ trachomatis are the most common pathogens associated with male infertility [27-29]. However, our study not only demonstrated an overall high prevalence of $\mathrm{U}$ urealyticum (10.41\%), and $\mathrm{M}$ hominis $(20.83 \%)$ but also showed that they were co-infected in widespread among asymptomatic infertile and fertile males. During this study we also detected other STD which were Chlamydia trachomatis (6.25 percent), Neisseria gonorrhoeae (4.16\%), Human Papillomavirus (2.08\%) and Trichomonas vaginalis (2.1 percent).

In 2004 first national STI study in Pakistan was conducted in order to determine the disease burden of HIV and STIs. The study showed a high burden of STIs among women selling sex, with the following prevalence; T. pallidum 7\%, N. gonorrhoeae $12 \%$, C. trachomatis $11 \%$ and $\mathrm{T}$. vaginalis $19 \%$. The study also highlighted a low level of knowledge about AIDS among the women selling sex; $24 \%$ had never heard of AIDS and $26 \%$ had not used a condom with their recent sexual partner. In Pakistan, the terms HIV and AIDS are commonly used synonymously.

The importance of genital tract microorganisms as an etiologic factor in male infertility is still a controversial topic [30]. The purpose of this study was to determine the prevalence of several common sexually transmitted pathogens among male members of infertile couples. Asymptomatically infected individuals may carry lower amount of organisms [31]. Besides, real time PCR is easier and has higher sensitivity and specificity. Thus, real time PCR may be the technique of choice for bacterial detection and quantification in semen specimens of asymptomatic male partners. Our study demonstrated that C. trachomatis seems to be the less spread sexually transmitted pathogen among male partners of infertile couples.

Infertility caused by Chlamydia was a big problem for women, the infection can cause scarring in the Fallopian Tubes which can block them and mean that the egg and sperm have a reduced chance of meeting. The infection is less reported in men, it is a significant issue as it can reduce the production of sperm (by up to $80 \%$ ) and cause sperm fragmentation which means that the long tail of sperm may break and the genetic material of the sperm is not as tightly packed [32].

Untreated chlamydia infections can also cause NSU—Non specific urethritis in men, which means that you may experience pain in the genital region, discomfort when urinating and/or having sex. This can happen even if there are no symptoms in the early stages of infection.

It has also been reported that that C. trachomatis infection could affect sperm mitochondrial function. Caspase activity has been shown to be present in human sperm [33-35]. Furthermore, in infertile men a higher percentage of sperm with activated caspases was found, confirming the existence of a caspase-dependent apoptotic pathway in ejaculated human sperm [36, 37]. In the third part of our study, we studied the activation of caspase 3 in spermatozoa of infertile men. We noticed also a significant increase of caspase 3 activation in male partners of infertile couples with $\mathrm{C}$. trachomatis DNA in semen specimens in comparison to male partners of infertile couples without C. trachomatis DNA in semen specimens. So public health are concerned about chlamydia infections because of the effect if can have on fertility. This can damage individual's mental health and well 
being, and cost thousands of pounds in fertility treatment. Preventing the infection at an early stage is the key to reducing these later issues. Public health also wants to protect against the costs of treating NSU in the future.

During our study co-infection detected was $\mathrm{U}$ urealyticum and $\mathrm{M}$ hominis was 4.8 percent, $\mathrm{C}$. trachomatis and Neisseria gonorrhoeae was 2.1 percent as shown in figure 4. It was reported as co-infection which was important STD screening parameters. It has been reported that N. gonorrhoeae infection is asymptomatic and often mistaken as virginal or bladder infection and may progress to serious complications as skin pustules or petechial, septic arthritis, memingitis or endocarditis [38].

\section{Conclusions}

The results of our study demonstrate that the genital mycoplasmas and ureaplasmas seem to be widespread among male partners of infertile couples as previously reported in Tunisia. The study of the comparison of the semen parameters of infertile men with and without genital ureaplasmas and mycoplasmas has not shown any significant differences, apart from the sperm concentration in the infection of $M$. hominis and M. genitalium and sperm morphology in the infection of M. hominis. Our results also indicate that PCR-and Gene Flow through hybridization assay method provides a rapid and cost effective measure to detect human genital mycoplasmas, ureaplasmas, chlamydia trachomatis and neisseria gonorrhoeae which are useful for etiological and epidemiological studies of these pathogens. Little information was, however, available regarding the effect of these STI on the sperm quality, as well as their relationship with the leukocyte count. Therefore, it can be concluded that the screening of these species in routine semen analysis is not clinically relevant in our specific population. It should be restricted for men undergoing complete evaluation of infertility, genital infection and male partners from couples undergoing IVF.

\section{Reference}

[1] Haidl, G., Allam, J. P., and Schuppe, H. C. 2008. "Chronic Epididymitis: Impact on Semen Parameters and Therapeutic Options." Andrologia 40 (2): 92-6.

[2] Comhaire, F. H., De Kretser, D., and Farley, T. M. M. 1987. "Towards More Objectivity in the Management of Male Infertility." Int. J. Androl. 10: 1.

[3] Dohle, G., Colpi, G., Hargreave, T., Papp, G., Jungwirth, A., and Weidner, W. 2005. "EAU Guidelines on Male Infertility." Eur. Urol. 48 (5): 703-11.

[4] Pellati, D., Mylonakis, I., Bertoloni, G., Fiore, C., Andrisani, A., and Ambrosini, G., et al. 2008. "Genital Tract Infections and Infertility." Eur. J. Obstet. Gynecol. Reprod Biol. 140 (1): 3-11.

[5] Smith, W. B. I. I., Trost, L. W., Chen, Y., Rosenchans, A., and Hellstrom, W. T. G. 2014. "Male Infertility: A Complete Guide to Lifestyle and Environmental Factors." Chapter 9 Springer, Science, New York 127.

[6] American Urological Association. The Optimal Evaluation of the Infertile Male. AUA Best Practice Statement. Revised 2010.

[7] Agacfidan, A., and Kohl, P. 1999. "Sexually Transmitted Diseases (STDs) in the World." FEMS Immunol. Med. Microbiol. 24 (4): 431-5.

[8] Hashwani, S., Hiran, T., and Fatima, M. 1999. "Awareness of Sexually Transmitted Diseases in a Selected Sample in Karachi." J. Pak. Med. Assoc. 49: 161-4.

[9] WHO: Global Strategy for the prevention and control of Sexually Transmitted Infections: 2006-2015. Breaking the chain of transmission WHO; 2007.

[10] White, R. G., Orroth, K. K., and Glynn, J. R., et al. 2008. "Treating Curable Sexually Transmitted Infections to Prevent HIV in Africa: Still an Effective Control Strategy?" J. Acquir. Immune Defic Syndr 47 (3): 346-53.

[11] Sangani, P., Rutherford, G., and Wilkinson, D. 2004. "Population-based Interventions Forreducing Sexually Transmitted Infections, Including HIV Infection." Cochrane Database Syst. Rev. 2: CD001220.

[12] Cohen, M. S. 1998. "Sexually Transmitted Diseases Enhance HIV Transmission: no longer a Hypothesis." Lancet 351 (3): 5-7.

[13] Mohsin, S. K., Magnus, U., Shakila, Z., and Cecilia, S. L. 2011. "HIV, STI Prevalence and Risk Behaviours among Women Selling Sex in Lahore, Pakistan. Khan et al." BMC Infectious Diseases 11: 119.

[14] British Andrology Society. British Andrology society guidelines for the screening of semen donors for donor insemination 1999. Liesnard CA. Screening of semen donors for infectious diseases. Hum. Reprod 14: 1823-6.2. 


\section{"Flow-through" Hybridization Technology}

[15] "The American Society for Reproductive Medicine. Guidelines for Gamete and Embryo Donation." Hum. Reprod 13 (2): 12-24.3, 1998. Fertil Steril 70.

[16] Critser, J. K. 1998. "Current Status of Semen Banking in the USA." Hum. Reprod 13 (2): 55-67.5.

[17] Craig, J. M., Barratt, C. L., and Kinghorn, G. R. 1997. "Semen Donors and STD Screening." Genitourin. Med. 73: 280-3.6.

[18] Greenblatt, R. M., Handsfield, H. H., Sayers, M. H., and Holmes, K. K. 1986. "Screening Therapeutic Insemination Donors for Sexually Transmitted Diseases: Overview and Recommendations." Fertil Steril 46: 351-64.

[19] Mascola, L., and Guinan, M. E. 1986. "Screening to Reduce Transmission of Sexually Transmitted Diseases in Semen used for Artificial Insemination.” N. Engl. J. Med. 314: 1354-9.

[20] Paavonen, J., and Wolner-Hanssen, P. 1989. "Chlamydia Trachomatis: A Majorthreat to Reproduction." Hum Reprod 4: 111-24.

[21] Peeling, R. W., and Embree, J. 2005. "Screening for Sexually Transmitted Infection Pathogens in Semen Samples." Can J. Infect Dis. Med. Microbiol. 16 (2): 73-6.

[22] Centers for Disease Control and Prevention. Sexually Transmitted Disease Surveillance, 2003, Atlanta, GA: U.S. Department of Health and Human Services, Centers for Disease Control and Prevention, September 2004.

[23] Griffiths, P. D., Clark, D. A., and Emery, V. C. 2000. "Beta Herpes Viruses in Transplant Recipients." $J$. Antimicrob. Chemother. 45 (T3): 29-34.

[24] Maleki, S., Motamedi, H., Moosavian, S., and Shahbaziyan, M. 2013. "Frequency of Mycoplasma hominis and Ureaplasma urealyticum in Females with Urogenital Infections and Habitual Abortion History in Ahvaz, Iran; Using Multiplex PCR.” Jundishapur Journal of Microbiology 6 (6): 1-5.

[25] Keck, C., Gerber-Schafer, C., Clad, A., Wilhelm, C., and Breckwoldt, M. 1998. "Seminal Tract Infections: Impact on Male Fertility and Treatment Options." Hum Reprod Update 4 (6): 891-903.

[26] Friberg, J., and Gnarpe, H. 1974. "Mycoplasmas in Semen from Fertile and Infertile Men." Andrologia 6 (1): 45-52.
[27] Cengiz, T., Aydoganli, L., Baykam, M., Mungan, N. A., Tuncbilek, E., Dincer, M., Yakupoglu, K., and Akalin, Z. 1997. "Chlamydial Infections and Male Infertility." Int. Urol. Nephrol. 29 (6): 687-93.

[28] Abdulrazzak, A. A., and Bakr, S. S. 2000. "Role of Mycoplasma in Male Infertility." East Mediterr Health J. 6 (1): 149-55.

[29] Veznik, Z., Pospisil, L., Svecova, D., Zajicova, A., and Unzeitig, V. 2004. "Chlamydiae in the Ejaculate: Their Influence on the Quality and Morphology of Sperm." Acta Obstet. Gynecol. Scand. 83 (7): 656-60.

[30] Dejucq, N., and Jegou, B. 2001. "Viruses in the Mammalian Male Genital Tract and Their Effects on the Reproductive System." Microbiol. Mol. Biol. Rev. 65: 208-31.

[31] Witkin, S. S. 2002. "Immunological Aspects of Genital Chlamydia Infections." Best Pract. Res. Clin. Obstet. Gynaecol. 16: 865-74.

[32] Centers for Disease Control and Prevention. 2002 Sexually Transmitted Diseases Treatment Guidelines. MMWR Morb Mortal Wkly Rep. 51: 1-80.

[33] Paasch, U., Grunewald, S., Agarwal, A., and Glandera, H. J. 2004. "Activation Pattern of Caspases in Human Spermatozoa." Fertil Steril 81: 802-9.

[34] Villegas, J., Schulz, M., Soto, L., and Sanchez, R. 2005. "Bacteria Induce Expression of Apoptosis in Human Spermatozoa." Apoptosis 10: 105-10.

[35] Weng, S. L., Taylor, S. L., Morshedi, M., Schuffner, A., and Duran, E. H., et al. 2002. "Caspase Activity and Apoptotic Markers in Ejaculated Human Sperm." Mol. Hum. Reprod. 8: 984-91.

[36] Paasch, U., Grunewald, S., Fitzl, G., and Glander, H. J. 2003. "Deterioration of Plasma Membrane is Associated with Activated Caspases in Human Spermatozoa." $J$. Androl. 24: 246-52.

[37] Sellami, H., Znazen, A., Sellami, A., Mnif, H., and Louati, N., et al. 2014. "Molecular Detection of Chlamydia Trachomatis and Other Sexually Transmitted Bacteria in Semen of Male Partners of Infertile Couples in Tunisia: The Effect on Semen Parameters and Spermatozoa Apoptosis Markers." PLoS ONE 9 (7): 1-12.

[38] Moran, J. S. 2007. Gonorrhoea. BMJ Clinical Evidence, 1604. 